\title{
Neonatal occlusion by strangulation of the caeco- appendix in a type I omphalocele of aitken: rare case
}

\begin{abstract}
Summary
The omphalocele constitutes a embryofoetopathy which exposes the newborn to multiple complications including digestive one. We report the case of a newborn with Aitken type I omphalocele under tanning according to the Grob method having secondarily presented a neonatal occlusion. Surgical exploration found a tight neck of $4 \mathrm{~cm}$ with a sac containing the ascending colon and the strangulated caeco-appendix at the level of the neck. We proceed to the reduction of the handles. We also noted on exploration, an arrangement of the handles in an incomplete common mesentery. He underwent an appendectomy then a provision of the slender handles on the right and the colon on the left according to the LADD procedure with closure of the abdominal cavity in one step. The operative consequences were simple after a follow-up of 6 months.
\end{abstract}

Keywords: occlusion, caeco-appendix, omphalocele, common mesentery
Volume II Issue 4 - 202I

\author{
Guedenon MA,' Yassegoungbe MG,' Boris \\ MVLS GOGAN,' Metchihoungbe CS,' \\ Seto DM, ${ }^{3}$ Akokpe MHO,' Segbedji GGPS,' \\ Amoussou AM,' Fiogbe $M^{2}$ \\ 'Department of Pediatric Surgery, Lagoon Mother and Child \\ University Hospital Center (CHU-MEL), Benin \\ ${ }^{2}$ University Clinic of Pediatric Surgery, National Hospital and \\ University Center Hubert Koutoukou Maga, Benin \\ ${ }^{3}$ Department of Surgery, Allada Zone Hospital, Benin
}

\section{Correspondence: Boris MVLS GOGAN, Department of Pediatric Surgery, Lagoon Mother and Child University Hospital Center (CHU-MEL), Benin, Tel +229 96102445,}

Email goganboris10@gmail.com

Received: July 19, 2021 | Published: August 05, 2021

\section{Introduction}

Malformations of the anterior wall of the abdomen form a broad spectrum of birth defects that have in common a defective closure of the anterior wall of the abdomen. The omphaloceles constitute a defect of closure of the umbilical ring with exteriorization of the abdominal viscera covered by the translucent and a vascular amniotic membrane. ${ }^{1}$ It is an embryo foetopathy which is sometimes part of a chromosomal aberration or a polymal formative syndrome ${ }^{2}$ with a generally good prognosis. This anomaly can be fatal or expose the fetus and the new born to multiple complications which depend on the associated malformations, the early treatment, the size of the neck and the contents of the omphalocele. ${ }^{3}$ Our case was a minor form (Type I from Aïtken) under tanning, secondarily entered occlusion. This observation derives its originality from the cause of neonatal occlusion and the mal rotation associated with this pathology.

\section{Observation}

This is a newborn received at the first hour of life for malformation of the anterior abdominal wall with umbilical swelling containing abdominal viscera covered by a translucent membrane. The gestational anamnesis made it possible to note a pregnancy followed without notion of taking teratogenic drug and without other incident. Note that the antenatal ultrasound had not been performed since the 2nd and 3rd trimester of pregnancy. The delivery was eutocic at 35 WA +2 days with good adaptation to ectopic life and a birth weight of $2900 \mathrm{~g}$. The child had emitted meconium on the 2 nd day of life. The initial examination found a translucent mass measuring $7 \mathrm{~cm}$ by $6 \mathrm{~cm}$ in diameter containing handles, with the umbilical cord inserted at the top. The contents were not hepatic.

The management taken was the management of omphalocele by tanning according to Grob on an outpatient basis under antibiotic coverage. The consequences were favorable with the preservation of the transit until the 3rd day of life, but it appeared greenish vomiting then fecaloid after a free interval of 6 days.
The examination made it possible to objectify a general bad impression with an asthenic child; a subictere; an epigastric arch on a painless supple depressible abdomen with the omphalocele on the way to epidemization. On digital rectal examination, the rectal bulb was empty. The diagnosis of neonatal occlusion on omphalocele was made. The newborn was taken to the operating room for an exploratory laparotomy. Under general anesthesia plus orotracheal intubation, installation in the dorsal decubitus position, warmed newborn, a transverse supra-umbilical incision is made according to Bianchi. At the coeliotomy, we objective: a tight neck of $4 \mathrm{~cm}$ with a sac containing the ascending colon and the strangulated caecoappendix at the level of the neck (Figure 1). We proceed to the reduction of the handles. Exploration of the abdomen made it possible to note an incomplete common mesentery loop arrangement (Figure 2). An appendectomy is performed and then the loops are placed in the complete common mesentery according to the LADD procedure; closure of the abdominal cavity, shot by shot after resection of the hernial sac and closure of the neck.

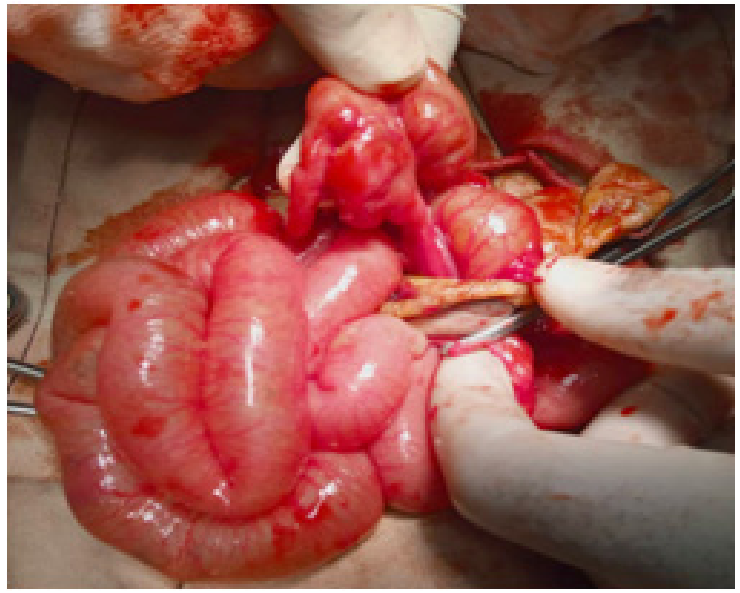

Figure I Strangulated caeco-appendix at the neck 


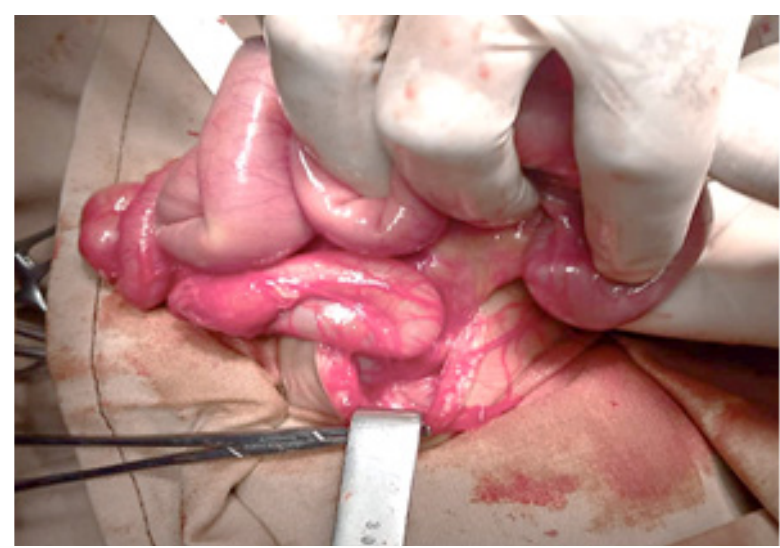

Figure 2 Presence of an incomplete common mesentery.

The operative consequences were simple with a resumption of transit on the 2 nd day and a well-tolerated diet without vomiting on the 4th day. The operative wound is well healed and the clinical examination is unremarkable after a follow-up of 6 months.

\section{Discussion}

Omphalocele is a frequent pathology with an annual incidence of 3 to 10 cases. ${ }^{4}$ In developed countries, the frequency is 1 case per 5000 births for small omphaloceles. ${ }^{4}$ Their frequency is lower compared to large omphaloceles. According to the literature, there is a risk of malformation in a pregnant woman aged 30 and even more after 35 years. The high risk would be between 35 and 40 years. ${ }^{5}$ The chromosomal abnormality in children of elderly mothers is classic. ${ }^{5}$ The Aïtken classification ${ }^{6}$ used is of prognostic and therapeutic interest. Type I omphaloceles have a better prognosis in countries with a high technical plateau. ${ }^{7}$ However, primary surgical closure remains the primary indication recommended in the literature. ${ }^{8}$ The the use of conservative methods is recommended in the treatment of type II omphaloceles. ${ }^{9}$ The surgical indication is systematic thanks to the progress of anesthesia and neonatal resuscitation as well as the development of antenatal diagnosis. ${ }^{10}$ In African countries and developing countries like ours, the therapeutic approach is different because of insufficient resuscitation and anesthesia resources. Grob's conservative tanning method is still used. In this context, the reduction and the cure of the residual eventration are carried out secondarily. This protocol was the one instituted in our case but the omphalocele proved to be complicated with intestinal obstruction. This complication was already described by Grob in 1963 and often present in type I. ${ }^{11}$ It is often due to strangulation of the handles by the neck which is less than $8 \mathrm{~cm}$. The most frequently encountered malformations in the literature are of cardiac, digestive, genitourinary and craniofacial origin. ${ }^{12}$ In our case, at the coeliotomy the malformation was digestive represented by an intestinal malrotation with an incomplete common mesentery. This malformative association is rare. The intestinal malrotation causes mobility of the colic loops allowing the caeco-appendage to be found in the omphalocele sac and to strangulate by the neck. The reduction was effective, as was the complete common mesentery arrangement after appendectomy using the LADD procedure. Surgery causes hydroelectrolyte and nutritional disturbances in the newborn which require parenteral nutrition with caloric intake of around $60 \mathrm{kcal} / \mathrm{kg} /$ day. The absence of neonatal resuscitation and parenteral nutrition means that only the context of neonatal occlusion (surgical emergency) allowed us to make the operative decision despite these difficulties.
This case allows us to review the management of uncomplicated type I omphaloceles in our context. The evolution being simple, it is urgent to provide the pediatric surgery departments with effective pediatric and neonatal resuscitation in order to proceed with surgery by primary closure of type I omphaloceles. The latter would make it possible to avoid complications, as in our clinical case. source of intestinal distress in most cases.

\section{Conclusion}

The omphalocele is a medium celosomy frequently associated with chromosomal aberrations or entering a polymalformative group as in the clinical case presented. This polymalformative association must remain in the minds of nursing staff for better monitoring and early detection of the malformation, guaranteeing a better prognosis.

\section{Acknowledgments}

None.

\section{Funding}

None.

\section{Conflicts of interest}

The authors have no conflicts of interest to declare.

\section{References}

1. Aigrain y, Lagausie P. Chirurgie des omphaloceles. In: Encycl. Med Chir. Elsevier, Paris, Surgical techniques. Digestive System. 1999;(10):40-142.

2. Agrain S, Napoli-Cocci, Lottiman H. Omphalocele and laparoschisis surgical treatment. Pediatric. 1993;347-351.

3. Ameh Ea, Dogo Pm, Nmadu Pt. Emergency neonatal surgery in a developing country. Pediatric Surgy Int. 2001;17(5-6):448-451.

4. Heider A. et al. Omphalocele: Outcomes of pregnancies in cases with normal karyotype. Am J Obst and Gynecol. 2004;190(1):135-141.

5. Chevrel Jp, Flament Jb. Treatment of elevations of the abdominal wall. Technical editions. Encycl Med Chir (Paris). Surgical Techniques Digestive System. 1995;40:165-110.

6. Brun M. Maugly LB, Rauch CF. Antenatal ultrasound diagnosis of Malformation of the anterior abdominal wall of the fetus. J. Radiol. 1998;79:1461-1468.

7. Lunzer H, Menardi G, Brezinka C. Long-term follow-up of children with prenatally diagnosed omphalocele andgastroschisis. J Matern Fetal Med. 2001;10(6):385-392.

8. Chevrel Jp, Flament Jb. Treatment of elevations of the abdominal wall. Technical editions. Encycl. Med. Chir. Surgical Techniques - Digestive System. 1995:40-165.

9. Gibbin C, Touch S, Broth RE, et al. Abdominal wall defect and congenial heart disease. Ultra sound obstet Gynecol. 2003;21 (4):334-337.

10. Kouamé BD, RG Dick, Ouattara O, et al. Therapeutic approaches of omphaloceles in developing countries: the experience of the Yopougon University Hospital, Abidjan, Ivory Coast, Bull. Soc Path Exot. 2003;96(4):302-305.

11. Grob M. Conservative treatment of exomphalos. Arch. 1963;5:148-150.

12. Angela L, Heider R, Strauss A, et al. Omphalocele: Clinical outcomes in cases with normal karyotypes. Am Jr of Obstet Gynecol. 2004;190(1):135141. 